\title{
FUSTr: a tool to find gene families under selection in transcriptomes
}

\author{
Timothy J Cole ${ }^{\text {Corresp., }} 1$, Michael S Brewer ${ }^{\text {Corresp. } 1}$ \\ ${ }^{1}$ Department of Biology, East Carolina University, Greenville, North Carolina, United States \\ Corresponding Authors: Timothy J Cole, Michael S Brewer \\ Email address: coleti16@students.ecu.edu, brewermi14@ecu.edu
}

Background: The recent proliferation of large amounts of biodiversity transcriptomic data has resulted in an ever-expanding need for scalable and user-friendly tools capable of answering large scale molecular evolution questions. FUSTr identifies gene families involved in the process of adaptation. This is a tool that finds genes in transcriptomic datasets under strong positive selection that automatically detects isoform designation patterns in transcriptome assemblies to maximize phylogenetic independence in downstream analysis.

Results: When applied to previously studied spider transcriptomic data as well as simulated data, FUSTr successfully grouped coding sequences into proper gene families as well as correctly identified those under strong positive selection in relatively little time.

Conclusions: FUSTr provides a useful tool for novice bioinformaticians to characterize the molecular evolution of organisms throughout the tree of life using large transcriptomic biodiversity datasets and can utilize multi-processor high-performance computational facilities. 
1 FUSTr: a tool to find gene Families Under Selection in Transcriptomes

2 T. Jeffrey Cole ${ }^{1, *}$, Michael S. Brewer ${ }^{1}$

$3{ }^{1}$ Department of Biology, East Carolina University, Greenville, NC 27858.

4 Correspondence: coleti16@students.ecu.edu

5

6 Abstract

7

9 answering large scale molecular evolution questions. FUSTr identifies gene families involved in

10 the process of adaptation. This is a tool that finds genes in transcriptomic datasets under strong

11 positive selection that automatically detects isoform designation patterns in transcriptome

12 assemblies to maximize phylogenetic independence in downstream analysis.

13 Results: When applied to previously studied spider transcriptomic data as well as

14 simulated data, FUSTr successfully grouped coding sequences into proper gene families as well

15 as correctly identified those under strong positive selection in relatively little time.

16 Conclusions: FUSTr provides a useful tool for novice bioinformaticians to characterize

17 the molecular evolution of organisms throughout the tree of life using large transcriptomic

18 biodiversity datasets and can utilize multi-processor high-performance computational facilities.

\section{Background}


Elucidating patterns and processes involved in the adaptive evolution of genes and

21 genomes of organisms is fundamental to understanding the vast phenotypic diversity found in

22 nature. Recent advances in RNA-Seq technologies have played a pivotal role in expanding

23 knowledge of molecular evolution through the generation of an abundance of protein coding

24 sequence data across all levels of biodiversity (Todd, Black \& Gemmell, 2016). In non-model

25 eukaryotic systems, transcriptomic experiments have become the de facto approach for functional

26 genomics in lieu of whole genome sequencing. This is due largely to lower costs, better targeting

27 of coding sequences, and enhanced exploration of posttranscriptional modifications and

28 differential gene expression (Wang, Gerstein \& Snyder, 2009). This influx of transcriptomic data

29 has resulted in a need for scalable tools capable of elucidating broad evolutionary patterns in

30 large biodiversity datasets.

31 Billions of years of evolutionary processes gave rise to remarkably complex genomic

32 architectures across the tree of life. Numerous speciation events along with frequent whole

33 genome duplications have given rise to a myriad of multigene families with varying roles in the

34 processes of adaptation (Benton, 2015). Grouping protein encoding genes into their respective

35 families de novo has remained a difficult task computationally. This typically entails homology

36 searches in large amino acid sequence similarity networks with graph partitioning algorithms to

37 cluster coding sequences into transitive groups (Andreev \& Racke, 2006). This is further

38 complicated in eukaryotic transcriptome datasets that contain several isoforms via alternative

39 splicing (Matlin, Clark \& Smith, 2005). Further exploration of Darwinian positive selection in

40 these families is also nontrivial, requiring robust Maximum Likelihood and Bayesian

41 phylogenetic approaches.

42 Here we present a fast tool for finding Families Under Selection in Transcriptomes

43 (FUSTr), to address the difficulties of characterizing molecular evolution in large-scale

44 transcriptomic datasets. FUStr can be used to classify selective regimes on homologous groups of 
45 phylogenetically independent coding sequences in transcriptomic datasets and has been verified

46 using large transcriptomic datasets and simulated datasets. The presented pipeline implements

47 simplified user experience with minimized third-party dependencies, in an environment robust to

48 breaking changes to maximize long-term reproducibility.

49 While FUSTr fills a novel niche among sequence evolution pipeline, a recent tool, VESPA

50 (Webb et al., 2017), performs several similar functions. Our tool differs in that it can accept de

51 novo transcriptome assemblies that are not predicted ORFs. VESPA requires nucleotide data to be

52 in complete coding frames, and does not filter isoforms or utilize transitive clustering to deal with

53 domain chaining. Additionally, VESPA makes use of slow maximum likelihood methods for tests

54 of selection and provides no information about purifying selection, whereas FUSTr utilizes a Fast

55 Unconstrained Bayesian Approximation (FUBAR) (Murrell et al., 2013) to analyze both

56 pervasive and purifying regimes of selection.

\section{Implementation}

58 FUSTr is written in Python with all data filtration, preparation steps, and command line

59 arguments/parameters for external programs contained in the workflow engine Snakemake

60 (Köster \& Rahmann, 2012). Snakemake allows FUSTr to operate on high performance

61 computational facilities, while also maintaining ease of reproducibility. FUSTr and all third-party

62 dependencies are distributed as a Docker container (Merkel, 2014). FUSTr contains ten

63 subroutines that takes transcriptome assembly FASTA formatted files from any number of taxa as

64 input and infers gene families that are either under diversifying or purifying selection. A graphical

65 overview of this workflow and parallelization scheme has been outlined in Fig. 1.

66

Data Preprocessing The first subroutine of FUSTr acts as a quality check step to ensure

67 input files are in valid FASTA format. Spurious special characters resulting from transferring text 
68 files between multiple operating system architectures are detected and removed to facilitate

69 downstream analysis.

70 Isoform detection Header patterns are analyzed to auto-detect whether the given assembly

71 includes isoforms by detecting naming convention redundancies commonly used in isoform

72 designations, in addition to comparing the header patterns to common assemblers such as Trinity

73 de novo assemblies (Haas et al., 2013) and Cufflinks reference genome guided assemblies

74 (Trapnell et al., 2014).

Gene prediction Coding sequences are extracted from transcripts using Transdecoder

v3.0.1 (Haas et al., 2013). Transdecoder predicts Open Reading Frames (ORFs) using likelihood-

77 based approaches. A single best ORFs for each transcript with predicted coding sequence is

78 extracted providing nucleotide coding sequences (CDS) and complementary amino acid

79 sequences. This facilitates further analyses requiring codon level sequences while using the more

80 informative amino acid sequences for homology inferences and multiple sequence alignments. If

81 the data contain several isoforms of the same gene, at this point only the longest isoform is kept

82 for further analysis to ensure phylogenetic independence. The user may customize the use of

83 Transdecoder by changing minimum coding sequence length (default: 30 codons) or strand-

84 specificity (default: off). Users also have the option to only retain ORFs with homology to known

85 proteins through a BLAST search against Uniref90 or Swissprot in addition to searching PFAM

86 to identify common protein domains.

87 Homology search All coding sequences are assigned a unique identifier and then

88 concatenated into one FASTA file. Homology of peptide sequences is assessed via BLASTP

89 acceleration through DIAMOND (v.0.9.10) with an e-value cutoff of $10^{-5}$.

90 Gene Family inference The resulting homology network is grouped into putative gene

91 families using transitive clustering with $\operatorname{SiLiX}$ v.1.2.11, which is faster and has better memory

92 allocation than other clustering algorithms such as MCL, and greatly reduces the problem of 
93 domain chaining (Miele, Penel \& Duret, 2011). Sequences are only added to a family with $35 \%$

94 minimum identity, $90 \%$ minimum overlap, with minimum length to accept partial sequences in

95 families as 100 amino acids, and minimum overlap to accept partial sequences of 50\%. These are

96 the optimal configurations of SiLiX (Bernardes et al., 2015), but the user is free to configure

97 these options.

98 Multiple sequence alignment and phylogenetic reconstruction Multiple amino acid

99 sequence alignments of each family are then generated using the appropriate algorithm

100 automatically detected using MAFFT v7.221 (Katoh \& Standley, 2013). Spurious columns in

101 alignments are removed with Trimal v1.4.1's gappyout algorithm (Capella-Gutiérrez \& Silla-

102 Martínez, 2009). Phylogenetic reconstruction of each family's untrimmed protein multiple

103 sequence alignment using FastTree v2.1.9 (Price, Dehal \& Arkin, 2010). Trimmed multiple

104 sequence codon alignments are then generated by reverse translation of the amino acid alignment

105 using the CDS sequences.

106 Tests for selective regimes Families containing at least 15 sequences have the necessary

107 statistical power for tests of adaptive evolution (Wong et al., 2004). Tests of pervasive positive

108 selection at site specific amino acid level are implemented with FUBAR (Murrell et al., 2013).

109 Unlike codeml, FUBAR allows for tests of both positive and negative selection using an ultra-fast

110 Markov chain Monte Carlo routine that averages over numerous predefined site-classes. When

111 compared to codeml, FUBAR performs as much as 100 times faster (Murrell et al, 2013). Default

112 settings for FUBAR, as used in FUSTr, include twenty grid points per dimension, five chains of

113 length 2,000,000, with the first 1,000,000 used as burn-in, 100 samples drawn from each chain,

114 and concentration parameter of the Dirichlet prior set to 0.5 .

115 Users have the option to also run tests for pervasive selection using the much slower

116 CODEML v4.9 (Yang, 2007) with the codon alignments and inferred phylogeny. Log-likelihood

117 values of codon substitution models that allow positive selection are then compared to respective 
118 nested models not allowing positive selection (M0/M3, M1a/M2a, M7/M8, M8a/M8), Bayes

119 Empirical Bayes (BEB) analysis then determines posterior probabilities that the ratio of 120 nonsynonymous to synonymous substitutions $\left(\mathrm{d}_{\mathrm{N}} / \mathrm{d}_{\mathrm{S}}\right)$ exceeds one for individual amino acid sites.

121 Final output and results The final output is a summary file describing what gene families

122 were detected, and those that are under strong selection and the average $d_{N} / d_{S}$ per family. A CSV

123 file for each family under selection is generated giving the following details per codon position of

124 the family alignment : alpha mean posterior synonymous substitution rate at a site; beta mean

125 posterior non-synonymous substitution rate at a site; mean posterior beta-alpha; posterior

126 probability of negative selection at a site; posterior probability of positive selection at a site;

127 Empiricial Bayes Factor for positive selection at a site; potential scale reduction factor; and

128 estimated effective sample site for the probability that beta exceeds alpha.

\section{Validation}

130 We tested FUSTr on six published whole body transcriptome sequences from an adaptive

131 radiation of Hawaiian Tetragnatha spiders (NCBI Short Read Archive Assesion numbers: 132 SRX612486, SRX612485, SRX612477, SRX612466, SRX559940, SRX559918) assembled 133 using the same methods from the original publication (Brewer et al., 2015). Spider genomes 134 contain numerous gene duplications lending to gene family rich transcriptomes. Additionally, this 135 adaptive radiation has been shown to facilitate strong, positive, sequence-level selection in these 136 transcriptomes (Brewer et al., 2015). This dataset provides an ideal case use for FUSTr. A total of 273,221 transcripts from all six Tetragnatha samples were provided as input for 138 FUSTr, a total of 4,258 isoforms were removed leaving 159,464 coding sequences for analysis

139 after gene prediction. The entire analysis ran in 13.7 core hours, completing within an hour when 140 executed on a 24-core server. Time of completion and memory usage for each of FUSTr's 
141 subroutines performance in this analysis is reported in Table 1. FUSTr recovered 134 families

142 containing at least 15 sequences, of these 46 families contained sites under pervasive positive 143 selection while all families also contained sites under strong purifying selection. This can be 144 contrasted to the analysis by Brewer et al. (2015) which found 2,647 one-to-one six-member 145 orthologous loci (one ortholog per each of the same samples), with 65 loci receiving positive 146 selection based on branch-specific analysis. The original analysis did not allow paralogs whereas

147 FUSTr does not reconstruct one-to-one orthogroups but entire putative gene families, and the 148 selection analysis utilized by FUSTr is site-specific and not branch-specific. Thus, it is not 149 expected that the results from FUSTr would perfectly match up with the original analysis, 150 however five of the 46 families FUSTr found to be under selection included loci from Brewer et 151 al.'s (2015) original 65 under selection based on branch-specific analysis.

152 The same 273,221 transcripts were entered as input for VESPA as a comparative analysis.

153 Because VESPA cannot filter Open Reading Frames in transcripts, it was unable to infer proper

154 coding sequences. In its first phase of cleaning input fasta files, 86,269 transcripts were wrongly 155 removed for having "internal stop codons" via improper reading frame inference, and 182,000 156 transcripts were removed due to "abnormal sequence length". Approximately $98 \%$ of the 157 transcripts were removed in the first phase of VESPA with no gene predictions, rendering further 158 analysis unnecessary for proper comparison of the performance of the two pipelines.

159 We further validated FUSTr by utilized coding sequences from simulated gene families 160 with predetermined selective regimes. We used EvolveAGene (Hall, 2007) on 3,000 random 161 coding sequences of a random length of 300-500 codons to generate gene families containing 16 162 sequences evolved along a symmetric phylogeny each with average branch lengths chosen 163 randomly between $0.01-0.20$ evolutionary units. Selective regimes with a selection modifier of 1643.0 were randomly chosen for each family so that a random $10 \%$ partition of the family receive 165 pervasive positive selection, purifying selection, or constant selection. All other settings for 
166 EvolveAGene were left as their defaults: the probability of accepting an insertion was set to the

167 default 0.1 , the probability of accepting a deletion defaulted to 0.025 , the probability of accepting

168 a replacement was left at 0.016 , no recombination was allowed. A visual schema for these

169 simulations can be found in Fig. 2.

170 The resulting 48,000 simulated sequences were used as input for FUSTr with

171 Transdecoder set to be strand-specific. FUSTr correctly recovered all 3,000 families, and all 975

172 that were randomly selected to undergo strong positive selection were correctly classified as

173 receiving pervasive positive selection. Additionally, the families selected to undergo purifying

174 selection were correctly classified, and families not selected receive constant selection were

175 classified as not having any specific sites undergoing purifying or pervasive positive selection.

176 Scripts for these simulations can be found at https://github.com/tijeco/FUSTr.

\section{Conclusions}

178 Current advances in RNA-seq technologies have allowed for a rapid proliferation of

179 transcriptomic datasets in numerous non-model study systems. It is currently the only tool

180 equipped to deal with the nuances of transcriptomic data, allowing for proper prediction of gene

181 sequences and isoform filtration. FUSTr provides a fast and useful tool for novice

182 bioinformaticians to detect gene families in transcriptomes under strong selection. Results from

183 this tool can provide information about candidate genes involved in the processes of adaptation,

184 in addition to contributing to functional genome annotation.

185 Availability: FUSTr is freely available under a GNU license and can be downloaded at 186 https://github.com/tijeco/FUSTr.

\section{Acknowledgements}


188 This work would not have been possible without XSEDE computational allocations

189 (BIO160060). We also thank Chris Cohen for editing this manuscript.

190 References

191 Andreev K, Racke H. 2006. Balanced Graph Partitioning. Theory of Computing Systems 39:929-

192 939. DOI: 10.1007/s00224-006-1350-7.

193 Benton R. 2015. Multigene Family Evolution: Perspectives from Insect Chemoreceptors. Trends in Ecology \& Evolution 30:590-600. DOI: 10.1016/j.tree.2015.07.009.

Bernardes JS., Vieira F.R., Costa L.M., Zaverucha G. 2015. Evaluation and improvements of clustering algorithms for detecting remote homologous protein families. $B M C$ Bioinformatics 16: 34 .

Brewer MS, Carter RA, Croucher PJP, and Gillespie, RG. 2015, Shifting habitats, morphology, and selective pressures: Developmental polyphenism in an adaptive radiation of Hawaiian spiders. Evolution, 69: 162-178. doi:10.1111/evo.12563

Capella-Gutiérrez S, Silla-Martínez JM. 2009. trimAl: a tool for automated alignment trimming in large-scale phylogenetic analyses. ....

Haas BJ, Papanicolaou A, Yassour M, Grabherr M, Blood PD, Bowden J, Couger MB, Eccles D, Li B, Lieber M, Macmanes MD, Ott M, Orvis J, Pochet N, Strozzi F, Weeks N, Westerman R, William T, Dewey CN, Henschel R, Leduc RD, Friedman N, Regev A. 2013. De novo transcript sequence reconstruction from RNA-seq using the Trinity platform for reference generation and analysis. Nature protocols 8:1494-512. DOI: 10.1038/nprot.2013.084.

Hall B. 2007. EvolveAGene 3: A DNA coding sequence evolution simulation program. Nature Precedings. DOI: 10.1038/npre.2007.1230.1.

Katoh K, Standley DM. 2013. MAFFT multiple sequence alignment software version 7: improvements in performance and usability. Molecular biology and evolution.

Köster J, Rahmann S. 2012. Snakemake - a scalable bioinformatics workflow engine. Bioinformatics 28:2520-2522.

Matlin A, Clark F, Smith C. 2005. Understanding alternative splicing: towards a cellular code. Nature Reviews Molecular Cell Biology:386-398. DOI: 10.1038/nrm1645.

Merkel D. 2014. Docker: lightweight linux containers for consistent development and deployment Linux J. 2392

Miele V, Penel S, Duret L. 2011. Ultra-fast sequence clustering from similarity networks with SiLiX. BMC Bioinformatics 12:1-9. DOI: 10.1186/1471-2105-12-116.

Murrell B, Moola S, Mabona A, Weighill T, Sheward D, Pond S, Scheffler K. 2013. FUBAR: A Fast, Unconstrained Bayesian AppRoximation for Inferring Selection. Molecular Biology and Evolution 30:1196-1205. DOI: 10.1093/molbev/mst030.

Price MN, Dehal PS, Arkin AP. 2010. FastTree 2-approximately maximum-likelihood trees for large alignments. PloS one. DOI: 10.1371/journal.pone.0009490.

Todd E, Black M, Gemmell N. 2016. The power and promise of RNA-seq in ecology and evolution. Molecular Ecology:1224-1241. DOI: 10.1111/mec.13526.

Trapnell C, Roberts A, Goff L, Pertea G, Kim D, Kelley D, Pimentel H, Salzberg S, Rinn J, Pachter L. 2014. Differential gene and transcript expression analysis of RNA-seq experiments with TopHat and Cufflinks. Nature Protocols:562-578. DOI: 10.1038/nprot.2012.016.

Wang Z, Gerstein M, Snyder M. 2009. RNA-Seq: a revolutionary tool for transcriptomics. Nature Reviews Genetics:57-63. DOI: 10.1038/nrg2484.

Webb, Andrew E., Thomas A. Walsh, and Mary J. O’Connell. 2017. VESPA: Very large-scale 
235 evolutionary and selective pressure analyses. PeerJ Computer Science 3: e118.

236 Wong W. S. W., Yang Z., Goldman N., \& Nielsen R. 2004. Accuracy and Power of Statistical Methods for Detecting Adaptive Evolution in Protein Coding Sequences and for Identifying Positively Selected Sites. Genetics, 168(2), 1041-1051. http://doi.org/10.1534/genetics.104.031153

240 Yang Z. 2007. PAML 4: Phylogenetic Analysis by Maximum Likelihood. Molecular Biology and 241 Evolution 24:1586-1591. DOI: 10.1093/molbev/msm088. 


\section{Figure 1 (on next page)}

Parallelization scheme and workflow of FUSTr.

Color coding denotes functional subroutines in the pipeline: preparation and open reading frame prediction (red); homology inferenece and gene family clustering (green); multiple sequence alignment, phylogenetics, and selection detection (brown); and model selection and reconciliation (blue). 


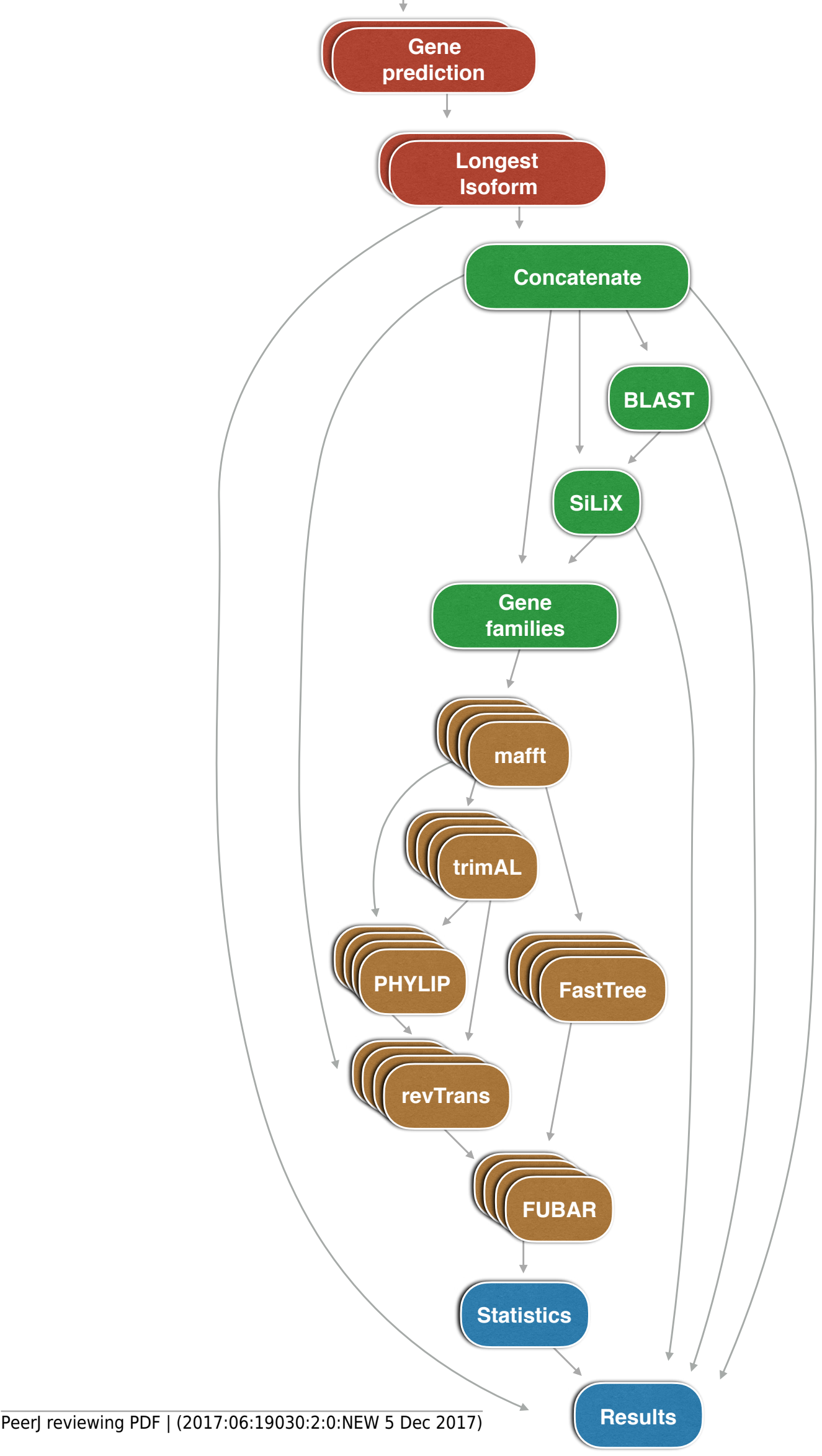


Figure 2 (on next page)

Schematice used for EvolveAGene.

Randomly generated sequence is evolved along a symmetric phylogeny of a given selective regime. 
1. Seed sequence (300-500 codons)

2. Evolve gene along 16 node phylogeny ( $\bar{x}$ branch length of $0.01-0.20$ )

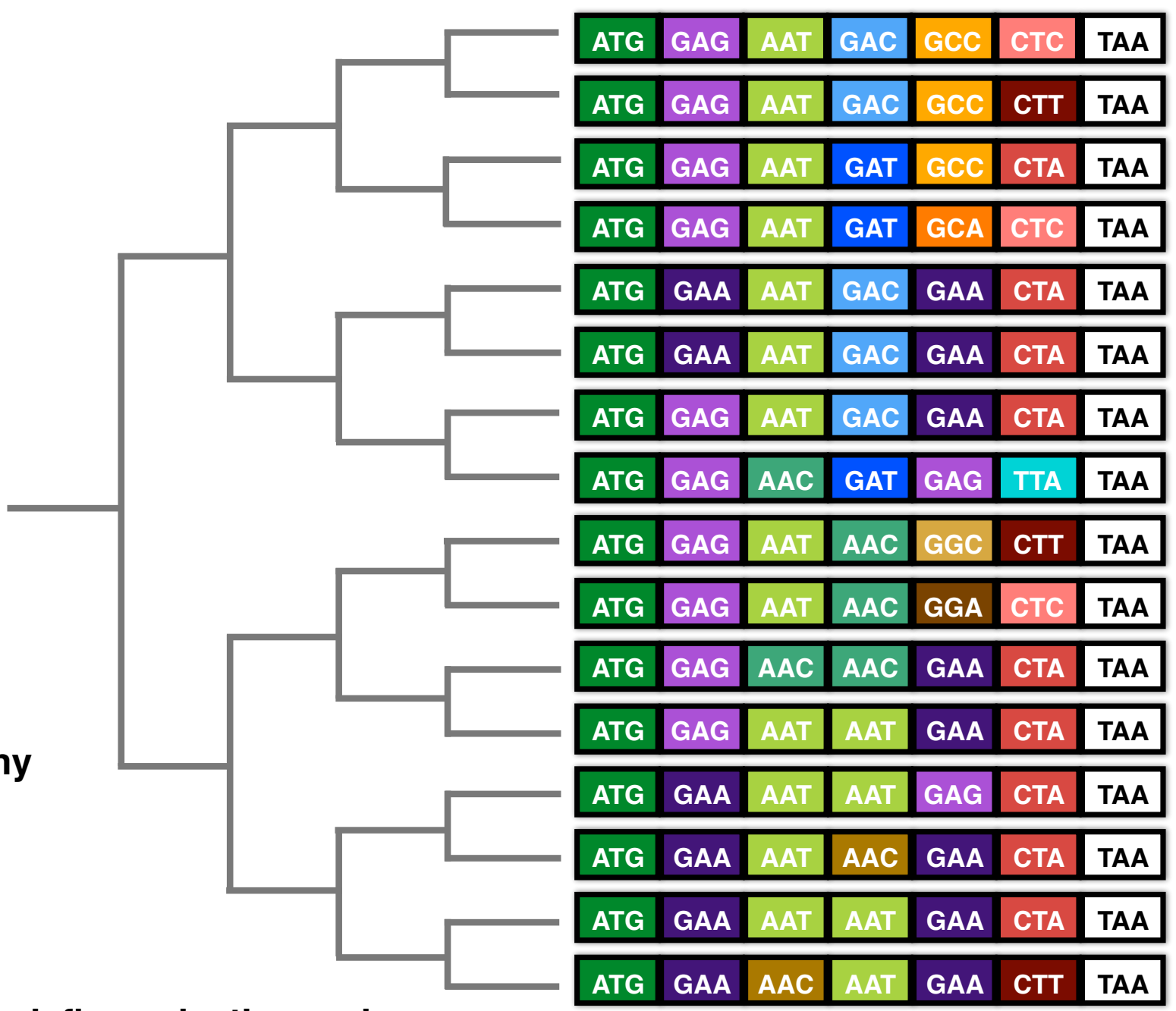

3. Predefine selective regime (positive, purifying, constant) across sequences 


\section{Table $\mathbf{1}$ (on next page)}

Benchmarks for each subroutines' time and memory used for the Tetragnatha transcriptome assembly analysis.

Red highlighted row represents subroutine consuming the most memory and time per task, blue highlighted row represents subroutine consuming the most memory and time in total. 
3

4

5

\begin{tabular}{lrrrrr} 
subroutine & tasks & $\begin{array}{r}\bar{x} \text { seconds } \\
\text { per task }\end{array}$ & $\begin{array}{r}\text { total } \\
\text { seconds }\end{array}$ & $\begin{array}{r}\text { x RAM per } \\
\text { task (MiB) }\end{array}$ & $\begin{array}{r}\text { total RAM } \\
(\mathrm{MiB})\end{array}$ \\
\hline Clean fastas & 6 & 1.40 & 8.38 & 46.5 & 278.9 \\
New headers & 6 & 1.65 & 9.90 & 43.6 & 261.5 \\
Long isoform & 6 & 0.512 & 3.07 & 51.5 & 309.13 \\
Transdecoder & 1 & $10,436.7$ & $10,436.7$ & $3,249.8$ & $3,249.8$ \\
Diamond & 1 & 32.1 & 32.1 & 234.0 & 234.0 \\
SiLiX & 1 & 4.51 & 4.51 & 22.8 & 22.8 \\
Mafft & 135 & 3.24 & 437.8 & 18.3 & $2,466.5$ \\
FastTree & 135 & 3.09 & 417.4 & 18.5 & $2,491.3$ \\
TrimAL & 135 & 1.87 & 252.2 & 17.9 & $2,415.6$ \\
FUBAR & 135 & 278.6 & $37,605.5$ & 28.8 & $3,886.2$
\end{tabular}

